\title{
Cell-free hemoglobin promotes primary graft dysfunction through oxidative lung endothelial injury
}

Ciara M. Shaver, ${ }^{1}$ Nancy Wickersham, ${ }^{1}$ J. Brennan McNeil, ${ }^{1}$ Hiromasa Nagata, ${ }^{2}$ Adam Miller, ${ }^{3}$ Stuart R. Landstreet, ${ }^{1}$ Jamie L. Kuck, ${ }^{1}$ Joshua M. Diamond, ${ }^{4}$ David J. Lederer, ${ }^{5}$ Steven M. Kawut, ${ }^{4}$ Scott M. Palmer, ${ }^{6}$ Keith M. Wille, ${ }^{7}$ Ann Weinacker, ${ }^{8}$ Vibha N. Lama, ${ }^{9}$ Maria M. Crespo, ${ }^{4}$ Jonathan B. Orens, ${ }^{10}$ Pali D. Shah, ${ }^{10}$ Chadi A. Hage, ${ }^{11}$ Edward Cantu III, ${ }^{12}$ Mary K. Porteous, ${ }^{4}$ Gundeep Dhillon, ${ }^{8}$ John McDyer, ${ }^{13}$ Julie A. Bastarache, ${ }^{1,14}$ Jason D. Christie, ${ }^{4}$ Lorraine B. Ware, ${ }^{1,14}$ and the Lung Transplant Outcomes Group (LTOG) ${ }^{15}$

'Division of Allergy, Pulmonary, and Critical Care Medicine, Department of Medicine, Vanderbilt University Medical Center, Nashville, Tennessee, USA. Department of Anesthesiology, Keio University School of Medicine, Tokyo, Japan. ${ }^{3}$ Tennessee Donor Services, Nashville, Tennessee, USA. ${ }^{4}$ Division of Pulmonary and Critical Care Medicine, Department of Medicine, Perelman School of Medicine at the University of Pennsylvania, Philadelphia, Pennsylvania, USA. ${ }^{5}$ Division of Pulmonary, Allergy, and Critical Care Medicine, Columbia University School of Medicine, New York, New York, USA. ${ }^{6}$ Division of Pulmonary and Critical Care Medicine, Duke University Medical Center, Durham, North Carolina, USA. ${ }^{7}$ Division of Pulmonary and Critical Care Medicine, University of Alabama at Birmingham, Birmingham, Alabama, USA.

${ }^{8}$ Division of Pulmonary and Critical Care Medicine, Stanford University Medical Center, Palo Alto, California, USA. ${ }^{9}$ Division of Pulmonary and Critical Care Medicine, University of Michigan Medical Center, Ann Arbor, Michigan, USA.

${ }^{10}$ Division of Pulmonary and Critical Care Medicine, Johns Hopkins University Medical Center, Baltimore, Maryland, USA. "Division of Pulmonary, Allergy, Critical Care, and Occupational Medicine, Indiana University School of Medicine, Indianapolis, Indiana, USA. ${ }^{12}$ Division of Cardiovascular Surgery, Perelman School of Medicine at the University of Pennsylvania, Philadelphia, Pennsylvania, USA. ${ }^{33}$ Division of Pulmonary, Allergy, and Critical Care, University of Pittsburgh, Pittsburgh, Pennsylvania, USA. ${ }^{14}$ Department of Pathology, Microbiology, and Immunology, Vanderbilt University Medical Center, Nashville, Tennessee, USA. ${ }^{15}$ The LTOG is detailed in the Supplemental Acknowledgments.

Primary graft dysfunction (PCD) is acute lung injury within 72 hours of lung transplantation. We hypothesized that cell-free hemoglobin (CFH) contributes to PGD by increasing lung microvascular permeability and tested this in patients, ex vivo human lungs, and cultured human lung microvascular endothelial cells. In a nested case control study of 40 patients with severe PCD at 72 hours and 80 matched controls without PGD, elevated preoperative CFH was independently associated with increased PCD risk (odds ratio $[\mathrm{OR}] 2.75,95 \% \mathrm{Cl}, 1.23-6.16, P=0.014$ ). The effect of CFH on PCD was magnified by reperfusion fraction of inspired oxygen $\left(\mathrm{FiO}_{2}\right) \geq 0.40$ (OR 3.41, $P=0.031$ ). Isolated perfused human lungs exposed to intravascular CFH (100 mg/dl) developed increased vascular permeability as measured by lung weight (CFH $14.4 \%$ vs. control $0.65 \%, P=$ 0.047 ) and extravasation of Evans blue-labeled albumin dye (EBD) into the airspace $(P=0.027)$. CFH (1 mg/dl) also increased paracellular permeability of human pulmonary microvascular endothelial cell monolayers (hPMVECs). Hyperoxia $\left(\mathrm{FiO}_{2}=0.95\right)$ increased human lung and hPMVEC permeability compared with normoxia $\left(\mathrm{FiO}_{2}=0.21\right)$. Treatment with acetaminophen $(15 \mu \mathrm{g} / \mathrm{ml})$, a specific hemoprotein reductant, prevented CFH-dependent permeability in human lungs $(P=$ 0.046 ) and hPMVECs ( $P=0.037)$. In summary, CFH may mediate PGD through oxidative effects on microvascular permeability, which are augmented by hyperoxia and abrogated by acetaminophen.

research grant support from Boehringe

Ingelheim. DJL is a consultant for

ImmuneWorks.

Submitted: November 9, 2017

Accepted: December 19, 2017

Published: January 25, 2018

Reference information:

JCI Insight. 2018;3(2):e98546.

https://doi.org/10.1172/jci.

insight.98546.

\section{Introduction}

Primary graft dysfunction (PGD) is a form of acute lung injury that is characterized by hypoxemia, pulmonary edema, and lung inflammation that develops within the first 72 hours after lung transplantation $(1,2)$. PGD is the most common cause of short-term mortality after lung transplantation and also con- 
tributes to the development of chronic lung allograft dysfunction (2). A variety of clinical risk factors for PGD have been identified with contributions from the donor (3-7), the recipient $(3-5,7-10)$, and operative variables $(4,5,8,11)$. In addition, a number of biomarkers have been associated with increased risk of PGD, including markers of innate and adaptive immune activation, epithelial and endothelial injury, coagulation, vascular permeability, and lipid peroxidation $(2,12-27)$. Despite identification of these clinical and biomarker predictors of PGD, the mechanisms leading to PGD are not well understood, and there are no specific therapeutic interventions for PGD.

Cell-free hemoglobin $(\mathrm{CFH})$ is a potent proinflammatory oxidant that accumulates in the circulation in clinical conditions associated with increased RBC fragility, such as sepsis or sickle cell disease, as a result of $\mathrm{RBC}$ shearing during cardiopulmonary bypass or hemodialysis, or during storage of RBCs. When CFH is released into the extracellular space, it can be oxidized and drive oxidant-mediated modification of proteins and lipids (28). Elevated levels of $\mathrm{CFH}$ have been associated with poor clinical outcomes in a variety of clinical conditions. For example, in sepsis, elevated levels of circulating $\mathrm{CFH}$ are associated with increased mortality and organ dysfunction (29). In the airspace, elevated levels of CFH are associated with lung epithelial injury and disruption of the alveolar-capillary barrier $(30,31)$. Whether $\mathrm{CFH}$ contributes to the pathogenesis of PGD is unknown. However, the known association of PGD with cardiopulmonary bypass (5), pulmonary hypertension (5), and lipid peroxidation (27) — risk factors that have also been associated with elevated CFH (29, 32-34) - suggests a potential mechanistic link. Based on these data, we hypothesized that CFH may play a mechanistic role in the development of PGD via effects on vascular permeability.

It also may be feasible to specifically target CFH-mediated oxidative injury for therapeutic benefit. Based on structural homology between the heme moiety of CFH and the peroxidase moiety of cyclooxygenase, the cyclooxygenase inhibitor acetaminophen (APAP) is a specific hemoprotein reductant that can reduce oxidized ferryl $\left(\mathrm{Fe}^{4+}\right) \mathrm{CFH}$ to less reactive $\mathrm{CFH}$ species, reducing the potential for $\mathrm{CFH}$-mediated lipid peroxidation (35). APAP prevented hemoprotein-induced renal injury through reduction of oxidized myoglobin in a rat model of rhabdomyolysis (35). In addition, in a clinical trial in critically ill patients with sepsis who had elevated CFH levels, administration of enteral APAP at standard FDA-approved doses resulted in lower levels of plasma lipid peroxidation products and improved renal function (28). Whether APAP can limit development of PGD after lung transplantation has not been studied.

Based on these data, the current study had 3 goals. First, we tested the hypothesis that elevated perioperative levels of circulating $\mathrm{CFH}$ are associated with increased risk for PGD after lung transplantation. Second, we investigated whether $\mathrm{CFH}$ induces acute lung injury by increasing microvascular permeability in the isolated perfused human lung and in cultured human microvascular endothelial cells. Finally, we tested whether oxygen exacerbates or APAP attenuates the injurious effects of elevated CFH in these model systems.

\section{Results}

Patient characteristics. Patients with International Society for Heart and Lung Transplantation (ISHLT) grade $3 \mathrm{PGD}$ (partial pressure arterial oxygen $\left[\mathrm{PaO}_{2}\right] /$ fraction of inspired oxygen $\left[\mathrm{FiO}_{2}\right]<200$ with radiographic infiltrates in the lung allograft) $(1,2)$ at 72 hours after transplantation were frequency matched with control patients with no PGD in the 72 hours after lung transplant according to the underlying diagnosis and type of lung transplant (Table 1). Patients on preoperative extracorporeal support were excluded. Patients with PGD were more likely to have received cardiopulmonary bypass and more likely to have a donor with a smoking history. As expected, both 90-day and 1-year mortality were significantly higher in patients with PGD.

Perioperative trends in plasma $\mathrm{CFH}$. Unlike healthy subjects who have very low levels of circulating $\mathrm{CFH}$ ( $<5 \mathrm{mg} / \mathrm{dl}$ ) (36), CFH was elevated (>10 mg/dl) in plasma collected preoperatively in the majority (91\%) of lung transplant recipients (Figure 1A). The median level of CFH was $30 \mathrm{mg} / \mathrm{dl}$ (range 0-390 mg/dl). Plasma CFH increased within 6 hours of surgery and was downtrending by 24 hours after surgery. Levels were similar between patients with chronic obstructive versus interstitial lung disease at each time point (Supplemental Figure 1; supplemental material available online with this article; https://doi.org/10.1172/ jci.insight.98546DS1). Patients who received intraoperative cardiopulmonary bypass had marked increases in plasma CFH between preoperative and 6-hour postoperative measurements (Figure 1B). Patient characteristics aside from PGD frequency were similar across CFH groups (Supplemental Table 1).

Preoperative $\mathrm{CFH}$ is independently associated with increased risk of $P G D$. Preoperative $\mathrm{CFH}$ levels greater than or equal to the median of $30 \mathrm{mg} / \mathrm{dl}$ were associated with increased frequency of grade 3 PGD at 72 hours (Figure $2 \mathrm{~A}, 45 \%$ vs. $23 \%, P=0.013$ ). Postoperative $\mathrm{CFH}$ levels were not associated with risk of PGD 
Table 1. Patient characteristics

\begin{tabular}{|c|c|c|c|}
\hline Characteristic & PGD cases $(n=40)$ & Controls $(n=80)$ & $P$ value \\
\hline $\mathrm{Age}^{\mathrm{A}}$ & $55(47,61)$ & $56(52,61)$ & 0.84 \\
\hline Male ${ }^{B}$ & $27(68 \%)$ & $40(50 \%)$ & 0.06 \\
\hline Diagnosis $^{B}$ & & & 0.67 \\
\hline COPD & $16(40 \%)$ & 30 (38\%) & \\
\hline CF & $0(0 \%)$ & $2(2 \%)$ & \\
\hline Bilateral lung transplant ${ }^{\mathrm{B}}$ & $24(60 \%)$ & $50(63 \%)$ & 0.85 \\
\hline Ischemic time $(\mathrm{min})^{\mathrm{A}}$ & $243(212,268)$ & $234(196,268)$ & 0.54 \\
\hline Cardiopulmonary bypass $^{\mathrm{B}}$ & $22(55 \%)$ & $25(31 \%)$ & 0.01 \\
\hline mPAPA & $31.7(22.0,40.7)$ & $25.0(20.0,33.7)$ & 0.08 \\
\hline $\mathrm{pRBC}$ transfusion $(\mathrm{ml})^{\mathrm{A}}$ & $625(313,1,500)$ & $500(250,1,000)$ & 0.24 \\
\hline 1-year mortality ${ }^{\mathrm{B}}$ & $13(32.5 \%)$ & $9(11.3 \%)$ & 0.005 \\
\hline
\end{tabular}

Cases were identified as having grade 3 primary graft dysfunction (PGD) within 72 hours. Controls were matched for underlying diagnosis and procedural type. Data are shown as $n(\%)$ or median (25th percentile, 75th percentile). Comparisons were made by $\chi^{2}$ test (A) or Mann Whitney $U$ test (B) as

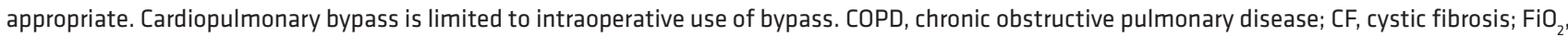
fraction of inspired oxygen; ILD, interstitial lung disease; mPAP, mean pulmonary artery pressure; pRBC, packed RBC.

(Figure 2B). In a univariate logistic regression model, a preoperative $\mathrm{CFH}$ level of $30 \mathrm{mg} / \mathrm{dl}$ or greater was associated with an odds ratio (OR) for PGD of 2.75 (95\% CI, 1.23-6.16, $P=0.014)$ (Table 2). This relationship persisted after individually controlling for other risk factors for PGD, including cardiopulmonary bypass, mean pulmonary artery pressure, donor smoking, BMI, sex, and reperfusion $\mathrm{FiO}_{2}$.

High reperfusion $\mathrm{FiO}_{2}$ increases the risk of $\mathrm{PGD}$. Because a higher $\mathrm{FiO}_{2}$ at reperfusion increased the association of lipid peroxidation with risk of PGD (27), we tested whether the association of $\mathrm{CFH}$ with risk of PGD was greater in those with higher reperfusion $\mathrm{FiO}_{2}(\geq 0.40)$. The median $\mathrm{FiO}_{2}$ was 0.25 (interquartile range [IQR]: $0.21-0.33)$ in the low $\mathrm{FiO}_{2}$ group $(n=46)$ and $0.74(\mathrm{IQR}: 0.58-0.95)$ in the high $\mathrm{FiO}_{2}$ group $(n=74)$. In patients with a high reperfusion $\mathrm{FiO}_{2}$, levels of $\mathrm{CFH}$ at or above the median were associated with increased risk of PGD (Figure 3). There was no statistically significant relationship between levels of $\mathrm{CFH}$ and risk of PGD in patients with low reperfusion $\mathrm{FiO}_{2}$. There was also no significant correlation between the concentration of $\mathrm{CFH}$ and reperfusion $\mathrm{FiO}_{2}$, suggesting that the impact of $\mathrm{FiO}_{2}$ on $\mathrm{PGD}$ risk is from modification of $\mathrm{CFH}$ oxidation rather than alteration in $\mathrm{CFH}$ release. We then performed multivariable logistic regression to further understand the relationship between $\mathrm{CFH}$, reper-

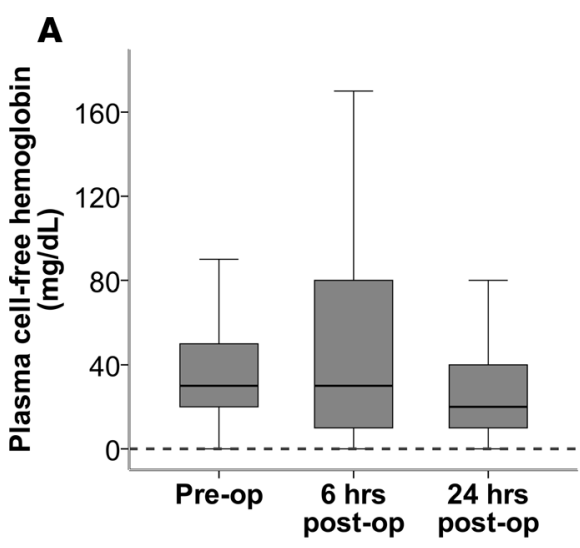

Figure 1. Plasma cell-free hemoglobin is elevated in lung transplant recipients. (A) Plasma cell-free hemoglobin (CFH) is detectable preoperatively as well as at 6 or 24 hours after lung transplantation. CFH was elevated in $91 \%, 97 \%$, and $85 \%$ of patients at each time point, respectively, $n=120$. The reference value of CFH in plasma in healthy patients is $<10 \mathrm{mg} / \mathrm{dl}$. (B) Use of cardiopulmonary bypass significantly increases CFH at 6 hours after transplant surgery, ${ }^{*} P<0.001$ by Mann Whitney $U$ test, $n=70$ without bypass, $n=44$ with bypass. 

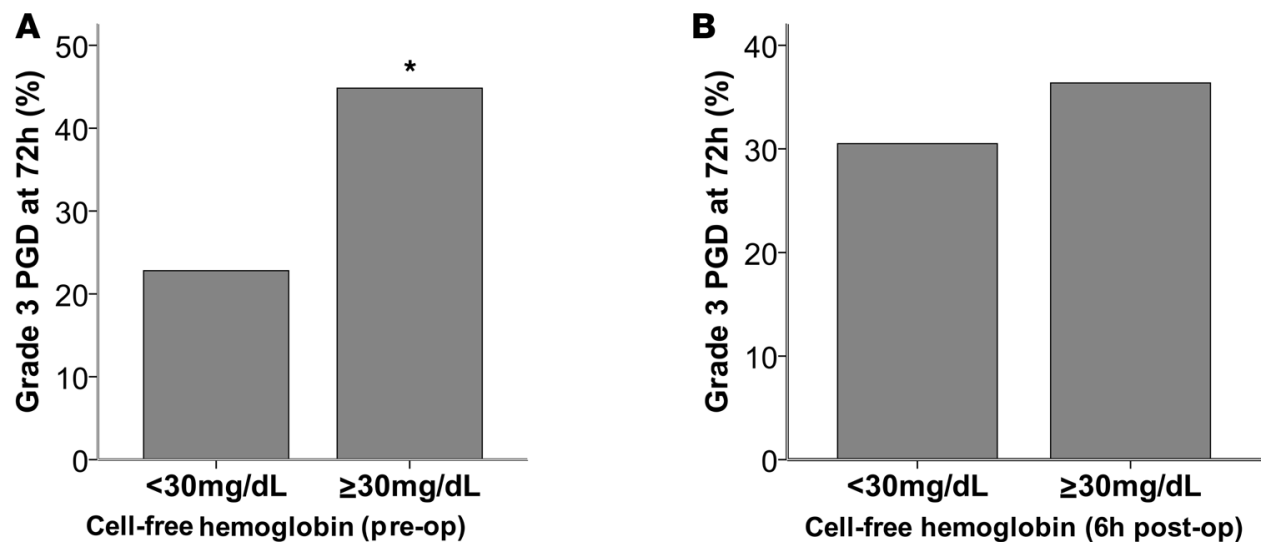

Figure 2. Preoperative plasma cell-free hemoglobin is associated with increased risk of primary graft dysfunction. (A) Patients with a plasma cell-free hemoglobin (CFH) level at or above the median (30 mg/dl) had a 1.9-fold increased risk of severe primary graft dysfunction (PGD) at 72 hours, ${ }^{*} P=0.013$ by $\chi^{2}$ analysis, $n=105$. (B) There was no association of CFH measured 6 hours postoperatively with risk of PGD, $P=0.51$ by $\chi^{2}$ analysis, $n=105$.

fusion $\mathrm{FiO}_{2}$, and PGD. Preoperative levels of $\mathrm{CFH}$ were independently associated with an increased risk of PGD only in patients with a reperfusion $\mathrm{FiO}_{2} \geq 0.40$ (high $\mathrm{FiO}_{2}, \mathrm{OR} 3.41,95 \% \mathrm{CI}, 1.12-10.42, P=$ 0.031; low $\mathrm{FiO}_{2}$, OR 2.78, 95\% CI, 0.54-14.38, $P=0.22$ ) (Table 3).

Circulating $\mathrm{CFH}$ increases lung vascular permeability in the isolated perfused human lung and cultured lung microvascular endothelial cells. The effect of $\mathrm{CFH}$ on lung vascular permeability was tested in isolated perfused human lungs obtained from deceased organ donors (Figure 4). Addition of a clinically relevant concentration of $\mathrm{CFH}(100 \mathrm{mg} / \mathrm{dl})$ to the lung perfusate increased vascular permeability in the presence of an $\mathrm{FiO}_{2}$ of 0.95 . Compared with control, lungs exposed to $\mathrm{CFH}$ in the perfusate had increasing lung weight over 2 hours ( $14.4 \%$ weight gain with $\mathrm{CFH}$ vs. $0.65 \%$ with control, $P=0.047$ ) (Figure $5 \mathrm{~A}$ ), an index of pulmonary edema formation. This was accompanied by increased extravasation of Evans blue-labeled albumin dye (EBD) from the perfusate into the alveolar space, a direct measure of vascular permeability (Figure 5B). Exposure of a tight monolayer of cultured human pulmonary microvascular endothelial cells ( $\mathrm{hPMVECs)} \mathrm{to} \mathrm{increasing} \mathrm{doses} \mathrm{of} \mathrm{CFH}$ for 24 hours increased paracellular permeability as assessed by a decrease in monolayer electrical resistance at $4,000 \mathrm{~Hz}$, showing a direct time-dependent effect of $\mathrm{CFH}$ on microvascular endothelial monolayer permeability (Figure 6).

Hyperoxia augments the effects of $\mathrm{CFH}$ on vascular permeability. Because of the association of reperfusion $\mathrm{FiO}_{2}$ with risk of PGD in our patient population, we next tested whether $\mathrm{FiO}_{2}$ affected $\mathrm{CFH}$-mediated changes in vascular permeability. For these studies, we compared the effect of $\mathrm{CFH}$ in the presence of an $\mathrm{FiO}_{2}$ of 0.95 to an $\mathrm{FiO}_{2}$ of 0.21 in paired lungs from the same donor to minimize the impact of donorto-donor variability. Lungs exposed to $\mathrm{CFH}$ in the presence of an $\mathrm{FiO}_{2}$ of 0.95 developed significantly

Table 2. Multivariable analysis of association between preoperative cell-free hemoglobin and primary graft dysfunction at 72 hours

\begin{tabular}{|c|c|c|c|}
\hline Variable & Odds ratio & $95 \% \mathrm{Cl}$ & $P$ value \\
\hline \multicolumn{4}{|l|}{ Unadjusted } \\
\hline Preoperative cell-free hemoglobin ( $\geq 30$ mg/dl) & 2.75 & $1.23-6.16$ & 0.014 \\
\hline \multicolumn{4}{|l|}{ Adjusted for } \\
\hline Cardiopulmonary bypass & 2.40 & $1.04-5.50$ & 0.039 \\
\hline Pulmonary artery pressure (mean) & 2.60 & $1.15-5.88$ & 0.021 \\
\hline Donor smoke exposure & 3.56 & $1.46-8.68$ & 0.005 \\
\hline $\mathrm{BMI}$ & 2.76 & $1.19-6.37$ & 0.018 \\
\hline Male sex & 2.89 & $1.26-6.66$ & 0.012 \\
\hline Reperfusion $\mathrm{FiO}_{2}$ & 2.88 & $1.27-6.51$ & 0.011 \\
\hline
\end{tabular}

The relationship between cell-free hemoglobin (CFH) and severe PGD, adjusted individually for each covariate is shown. CFH is measured in mg/dl in plasma. The referent group is patients with preoperative CFH below the median of $30 \mathrm{mg} / \mathrm{dl}$. PGD, primary graft dysfunction. 


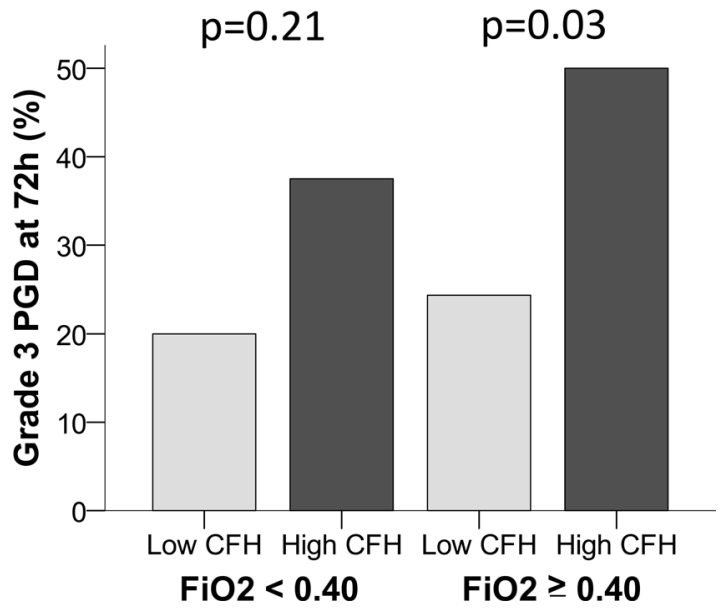

Figure 3. The association of cell-free hemoglobin with risk of primary graft dysfunction is modified by reperfusion $\mathrm{FiO}_{2}$ greater than $\mathbf{0 . 4 0}$. Primary graft dysfunction (PGD) is most common in patients with high cell-free hemoglobin (CFH) and high reperfusion $\mathrm{FiO}_{2}$. Comparisons were made between low $(<30 \mathrm{mg} / \mathrm{dl})$ and high ( $\geq 30$ $\mathrm{mg} / \mathrm{dl}$ ) CFH groups by $\chi^{2}$ testing. $n=105$.

more pulmonary edema as measured by weight gain (Figure 7A) and extravasation of EBD (Figure 7B). Prior to addition of $\mathrm{CFH}$ to perfusate, there was no significant difference in weight change between normoxic and hyperoxic conditions (normoxia, median [IQR] $-0.27 \%[-4.43 \%,-0.16 \%]$; hyperoxia, $-0.12 \%$ $[-2.67 \%, 0.06 \%], P=0.47$ by Mann Whitney $U$ test). Hyperoxia magnified CFH-dependent permeability changes in hPMVECs (Figure 7C). Cell viability of hPMVECs as measured by trypan blue exclusion was not affected by exposure to $\mathrm{CFH}$ or hyperoxia (data not shown).

APAP, a specific hemoprotein reductant, attenuates the effects of CFH on vascular permeability. Because of a structural similarity between the heme moiety of hemoglobin and the peroxidase moiety of cyclooxygenase, APAP specifically reduces oxidized $\mathrm{Fe}^{4+}$ hemoglobin to the less injurious reduced state $(35,37)$. Therefore, to determine whether the effects of hemoglobin on vascular permeability are attenuated by reduction of $\mathrm{CFH}$ into a less oxidized state, we next tested whether APAP could ameliorate $\mathrm{CFH}$-induced lung injury in paired human lungs. Addition of APAP at levels in the clinically therapeutic range $(15 \mu \mathrm{g} / \mathrm{ml})$ to the perfusate of the isolated human lung attenuated both $\mathrm{CFH}$-dependent lung weight gain (Figure 8A) and extravasation of EBD (Figure 8B). Prior to addition of $\mathrm{CFH}$ to perfusate, there was no significant difference in weight change between control and APAP ( $\mathrm{CFH}$ alone, median $[\mathrm{IQR}]-0.92 \%[-2.55 \%, 1.05 \%]$; CFH+APAP, $0.35 \%[-1.12 \%, 0.82 \%], P=0.34$ by Mann Whitney $U$ test), indicating that, in the absence of $\mathrm{CFH}$, APAP had no effect on lung weight. APAP prevented $\mathrm{CFH}$-induced increases in paracellular permeability of hPMVECs as measured by monolayer resistance (Figure 8C).

\section{Discussion}

PGD is a major cause of morbidity and mortality after lung transplantation. Taken together, the clinical and experimental data in this study support a mechanistic contribution of CFH to the development of PGD. The primary finding is that elevated preoperative levels of plasma $\mathrm{CFH}$ are independently associated with increased risk of PGD in lung transplant recipients. Mechanistically, addition of $\mathrm{CFH}$ to the perfusate in the ex vivo perfused human lung increased vascular permeability and pulmonary edema formation. Exposure to $\mathrm{CFH}$ also increased

Table 3. Multivariable analysis of association between preoperative cell-free hemoglobin, reperfusion Fi02, and primary graft dysfunction at 72 hours

\begin{tabular}{lccc}
\hline \multicolumn{1}{c}{ Variable } & Odds ratio & 95\% Cl & P value \\
$\begin{array}{l}\text { Low reperfusion } \mathrm{FiO}_{2}(<0.40) \\
\quad \text { Preoperative cell-free hemoglobin }(>30 \mathrm{mg} / \mathrm{dl})\end{array}$ & 2.78 & $0.54-14.38$ & 0.22 \\
$\quad \begin{array}{ll}\text { High reperfusion } \mathrm{FiO}_{2}(\geq 0.40) \\
\quad \text { Preoperative cell-free hemoglobin }(\geq 30 \mathrm{mg} / \mathrm{dl})\end{array}$ & 3.41 & $1.12-10.42$ & 0.031
\end{tabular}

The relationship between cell-free hemoglobin (CFH) and severe PCD, adjusted for recipient diagnosis, procedure type, mean pulmonary arterial pressure, and use of cardiopulmonary bypass. CFH is measured in $\mathrm{mg} / \mathrm{dl}$ in plasma. The referent group is patients with preoperative CFH below the median of $30 \mathrm{mg} / \mathrm{dl}$. 


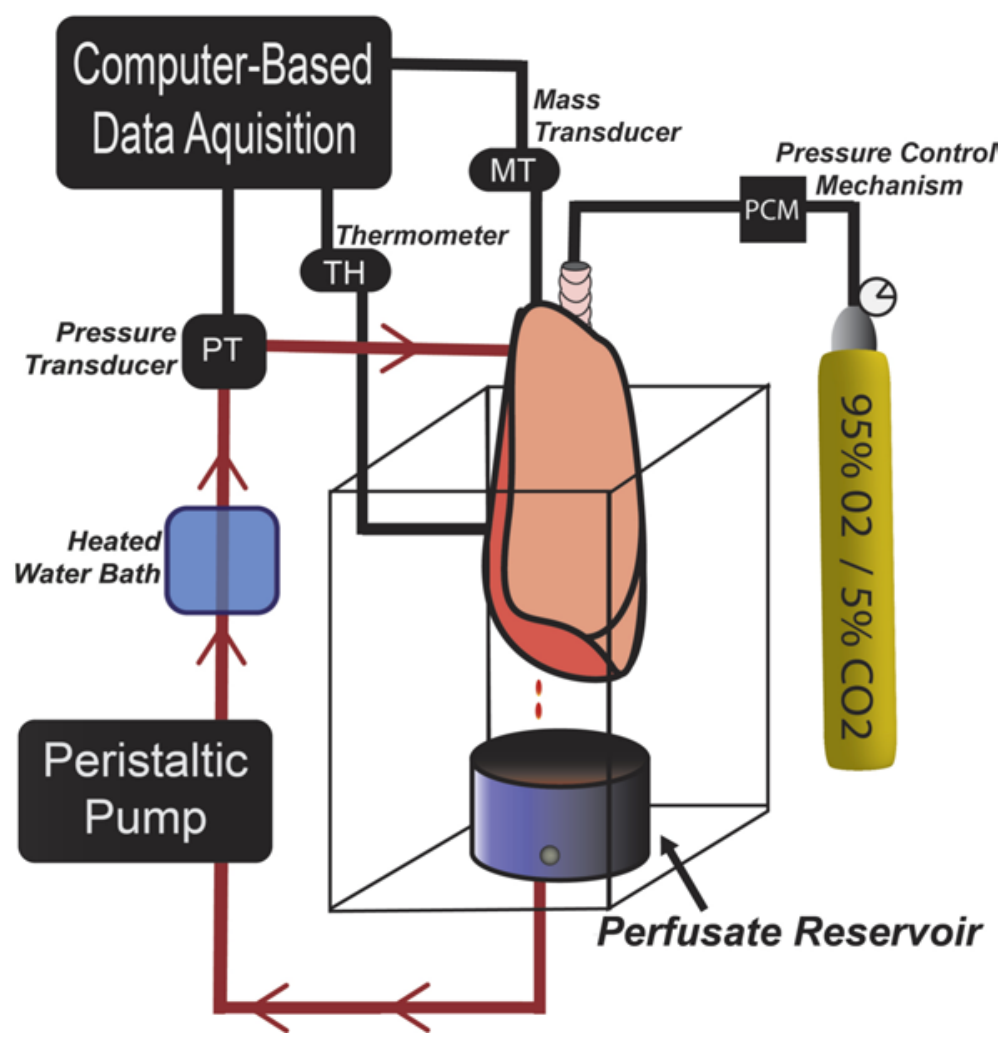

Figure 4. Schematic of ex vivo isolated perfused lung preparation. Lungs were suspended from a mass transducer and were continuously perfused at $37^{\circ} \mathrm{C}$ with DMEM containing $5 \%$ albumin and $16 \%(v / v)$ fresh whole human blood by roller pump to maintain a pulmonary artery pressure of $10-12 \mathrm{mmHg}$ with passive drainage of perfusate through an open left atrium $(53,54)$. Lungs were inflated to continuous positive end-expiratory pressure of $10 \mathrm{mmHg}$ with either an $\mathrm{FiO}_{2}$ of 0.95 or of 0.21 . The lung was suspended from a mass transducer for continuous measurement of lung weight as an index of pulmonary edema formation.

permeability in cultured pulmonary microvascular endothelial cell monolayers, demonstrating that $\mathrm{CFH}$ has direct effects on microvascular endothelial cell barrier function. Patients who received reperfusion $\mathrm{FiO}_{2}$ at or above 0.40 had increased risk of PGD compared with those with lower reperfusion $\mathrm{FiO}_{2}$. Similarly, the injurious effects of $\mathrm{CFH}$ were magnified in the presence of hyperoxia in isolated human lungs and in hPMVECs. Finally, the detrimental effects of $\mathrm{CFH}$ were attenuated by targeting the oxidation state of $\mathrm{CFH}$ with clinically relevant doses of APAP, a specific hemoprotein reductant. These findings identify $\mathrm{CFH}$ as a mediator of increased vascular permeability that contributes to the pathophysiology of PGD, and suggest that targeting oxidized CFH with the hemoprotein reductant APAP perioperatively could be a novel therapeutic strategy for prevention of PGD.

The current translational studies suggest that $\mathrm{CFH}$ contributes to increased microvascular permeability

A

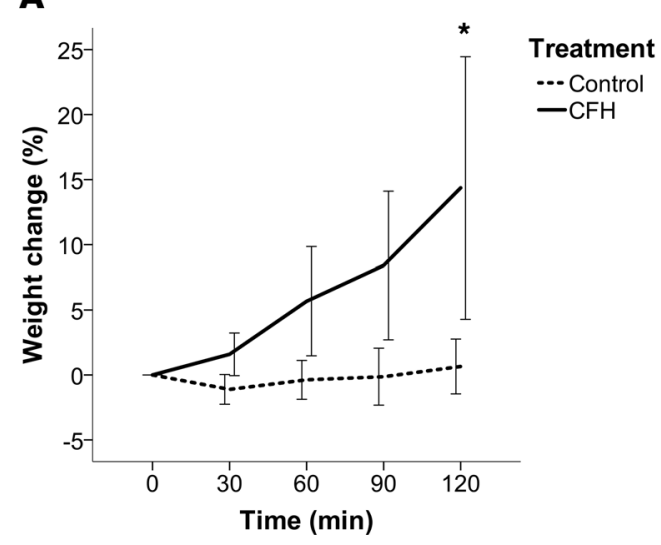

B

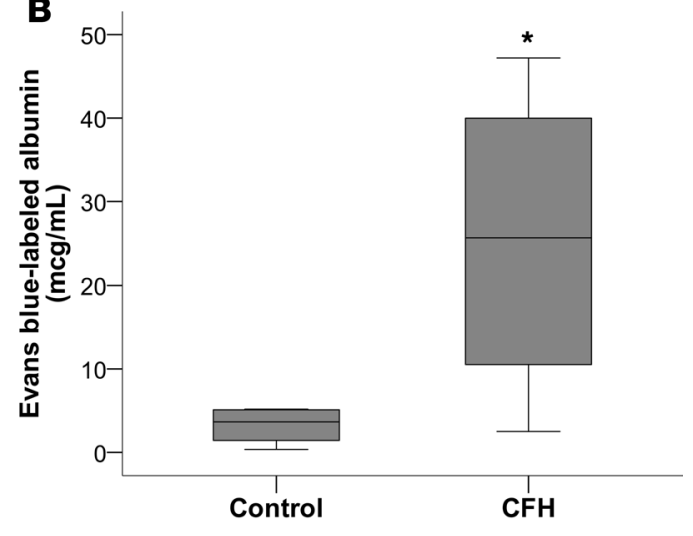

Figure 5. Cell-free hemoglobin increased pulmonary edema formation and vascular permeability in ex vivo isolated perfused lungs. (A) Cell-free hemoglobin (CFH) added to the perfusate $(100 \mathrm{mg} / \mathrm{dl})$ in the presence of hyperoxia $\left(\mathrm{FiO}_{2}=0.95\right)$ results in persistent weight gain over time, indicative of formation of pulmonary edema, $n=5$ per group, ${ }^{*} P=0.047 \mathrm{vs}$. control. (B) CFH increased vascular permeability as evidenced by extravasation of Evans blue-labeled albumin into bronchoalveolar lavage fluid, $n=5$ per group, ${ }^{*} P=0.027$ vs. control at 2 hours. Comparisons were made between control and CFH groups by Mann Whitney $U$ tests. 


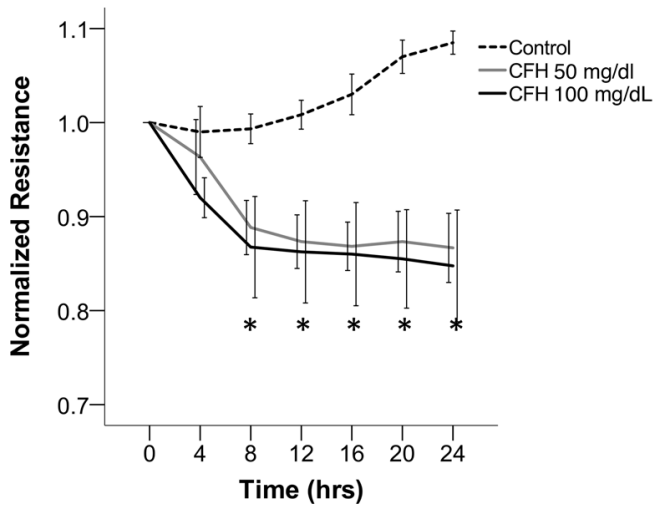

Figure 6. Cell-free hemoglobin increases pulmonary microvascular endothelial cell monolayer permeability. Cell-free hemoglobin (CFH) causes increased permeability as measured by a reduction in electrical resistance across the monolayer. Data are presented after normalization to the baseline resistance in each treatment chamber. ${ }^{*} P<0.05$ vs. control by Mann Whitney $U$ tests at each time point, $n=6$ per group.

in PGD. While other studies have reported associations between circulating $\mathrm{CFH}$ and alterations in endothelial function in models of atherosclerosis, malaria, and sickle cell disease (38-40), there have been few prior studies of the direct effects of $\mathrm{CFH}$ on microvascular endothelial permeability (41). Oxidized $\mathrm{CFH}$, which is present in atherosclerotic plaques, results in intercellular gap formation and increased permeability in cultured endothelial cell monolayers $(42,43)$. Hypoxia-responsive genes including HIF have also been implicated in hemoprotein-induced endothelial permeability in dermal microvascular endothelial cells (41). Another study showed that cross-linked CFH disrupted mesenteric permeability through actin cytoskeletal rearrangements and gap formation between intestinal endothelial cells (44). The data reported here support a potential mechanistic role for $\mathrm{CFH}$ in the pathogenesis of pulmonary microvascular permeability in PGD. Whether CFH impacts other known mediators of increased vascular permeability in this model, such as VEGF $(24,45,46)$, angiopoeitin-2 (23), or integrin $\alpha v \beta 5$ (47-49), will require further study.

In addition to being both a preoperative marker and a mediator of PGD, CFH can be targeted therapeutically. The rationale for a potential therapeutic effect of APAP is based on structural homology between the heme moiety of $\mathrm{CFH}$ and the peroxidase domain of cyclooxygenase, one of the molecular targets of APAP (35), a feature that is not present in other iron-containing proteins. APAP reduces the tyrosine radical that results from oxidation of $\mathrm{CFH}$ into its $\mathrm{Fe}^{4+}$ oxidation state (35). By this mechanism, APAP reduces the iron in the heme moiety of $\mathrm{CFH}$ from $\mathrm{Fe}^{4+}$ to $\mathrm{Fe}^{3+}$, thereby diminishing the capacity for $\mathrm{CFH}$ to drive lipid peroxidation. The benefit of this therapeutic approach has been demonstrated in a rat model of rhabdomyolysis where APAP administration mitigated renal injury induced by release of myoglobin, another hemoprotein that can be reduced by APAP (35). In a randomized placebo-controlled clinical trial in patients with severe sepsis, APAP decreased circulating lipid peroxidation products and reduced acute kidney injury in patients with elevated plasma CFH (28). In the current study, we demonstrated that clinically relevant doses of APAP, an inexpensive, safe, and widely available drug, limited $\mathrm{CFH}$-induced pulmonary edema formation and vascular permeability in both the isolated perfused human lung and cultured pulmonary microvascular endothelial cells. The association of preoperative $\mathrm{CFH}$ levels with PGD suggests that a treatment targeting $\mathrm{CFH}$ may be most effective if started prior to reperfusion. One approach would be to begin APAP administration in the lung recipient at the time of organ allocation or at the time of waitlisting. Together, these results provide compelling support for a future clinical trial of APAP for prevention or treatment of PGD.

The finding that preoperative $\mathrm{CFH}$ levels had a stronger association with PGD than postoperative $\mathrm{CFH}$ levels was unexpected and suggests that early exposure of the reperfused pulmonary vascular bed to high levels of CFH may be critical for the development of PGD. The striking relationship between high $\mathrm{CFH}$, high reperfusion $\mathrm{FiO}_{2}$, and increased risk of $\mathrm{PGD}$ suggests that hyperoxia at the time of reperfusion after implantation may potentiate the injurious effects of $\mathrm{CFH}$. This hypothesis is strongly supported by the finding that hyperoxia augments the detrimental effects of $\mathrm{CFH}$ on isolated human lungs and on endothelial cells. Another therapeutic approach that may attenuate the toxicity of preexisting circulating $\mathrm{CFH}$ in the transplant recipient would be to limit the reperfusion $\mathrm{FiO}_{2}$ to the lowest possible level required to achieve adequate oxygenation. In the current study, $62 \%$ of patients received a reperfusion $\mathrm{FiO}_{2} \geq 0.40$, suggesting that the majority of transplant recipients might benefit from limiting early hyperoxia. 


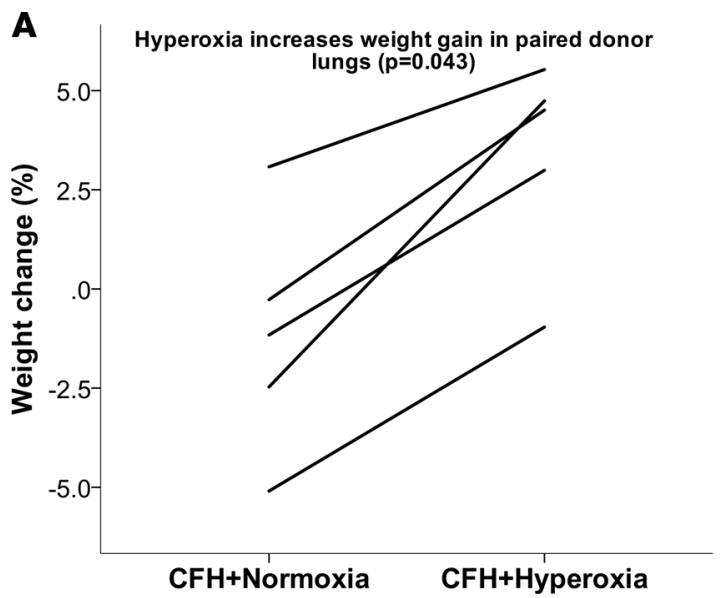

CFH+Normoxia CFH+Hyperoxia

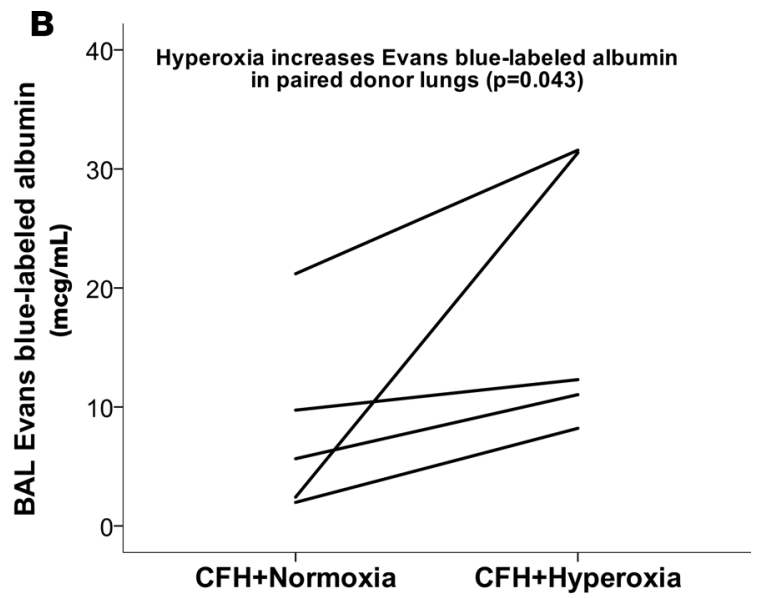

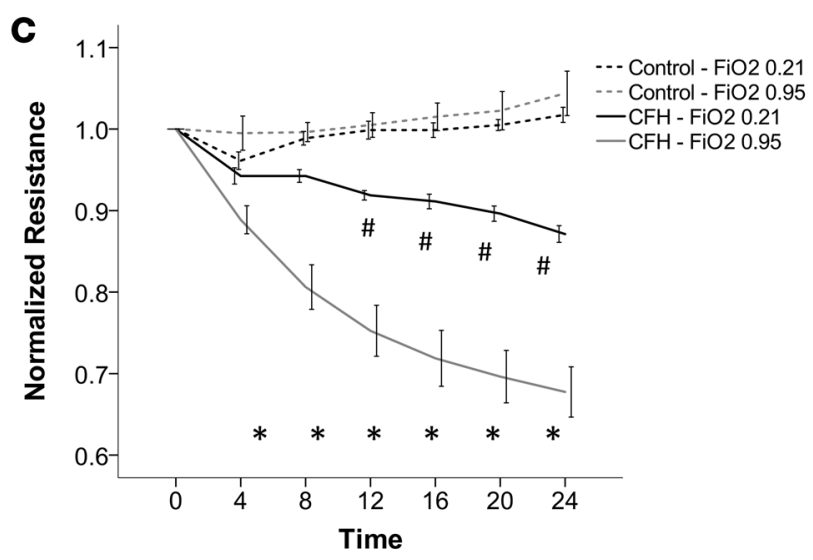

Figure 7. Hyperoxia exacerbates the toxicity of cell-free hemoglobin in the human lung and in human lung microvascular endothelial cells. (A) Ex vivo human isolated perfused lungs have greater weight gain over time after cell-free hemoglobin (CFH) exposure in the presence of hyperoxia. Each line connects lung weight change for paired donor lungs subjected to $\mathrm{CFH}$ in normoxia $\left(\mathrm{FiO}_{2} 0.21\right)$ and $\mathrm{CFH}$ in hyperoxia $(\mathrm{FiO}, 0.95)$. $n=5, P=0.043$ by Wilcoxon rank sum testing. (B) Hyperoxia exacerbates extravasation of Evans blue-labeled albumin into the airspace. $n=5, P=0.043$ by Wilcoxon rank sum testing. (C) Hyperoxia exacerbates CFH-induced permeability of cultured human pulmonary microvascular endothelial cells. $n=8$ per group, ${ }^{*} P<$ 0.05 vs. control $\mathrm{FiO}_{2} 0.95,{ }^{\#} P<0.05$ vs. control $\mathrm{FiO}_{2} 0.21$ by Mann Whitney $U$ test.

The explanation for the high preoperative levels of circulating $\mathrm{CFH}$ in lung transplant recipients is uncertain. One possibility is that patients with advanced obstructive or fibrotic lung disease develop elevated $\mathrm{CFH}$ due to chronic RBC shearing in diseased pulmonary microvasculature. Pulmonary arterial hypertension is associated with elevated $\mathrm{CFH}$ (33), and pulmonary hypertension due to advanced lung disease may share this association. Furthermore, chronic lung and systemic inflammation in the setting of end-stage lung disease might lead to a persistent oxidant-rich environment to trigger $\mathrm{CFH}$-mediated injury, in part due to depletion of protective antioxidants. Consistent with this concept, reduced levels of the antioxidants glutathione and protein-cys-SH were reported in blood of smokers with chronic obstructive pulmonary disease (COPD) compared with smokers without COPD (50). Further studies in large patient cohorts are needed to determine the mechanisms and consequences of elevated circulating $\mathrm{CFH}$ in patients with advanced lung disease.

This study has some limitations. Since the experimental studies only included lungs that were declined for transplantation, it is possible that preprocurement lung injury in the donor modified the impact of $\mathrm{CFH}$. However, the majority of procured donor lungs declined for transplantation had intact alveolar fluid clearance $(51,52)$, an indication that these donor lungs were physiologically intact. We attempted to minimize the effects of any preprocurement lung injury in 2 ways. First, to be included in the experimental protocol, each lung had to maintain a stable baseline weight for 30-60 minutes prior to the beginning of the experiment. This ensures that lungs have an intact microvascular barrier prior to the start of the experiment. Second, because of donor- 
A APAP reduces weight gain in paired donor lungs $(p=0.046)$

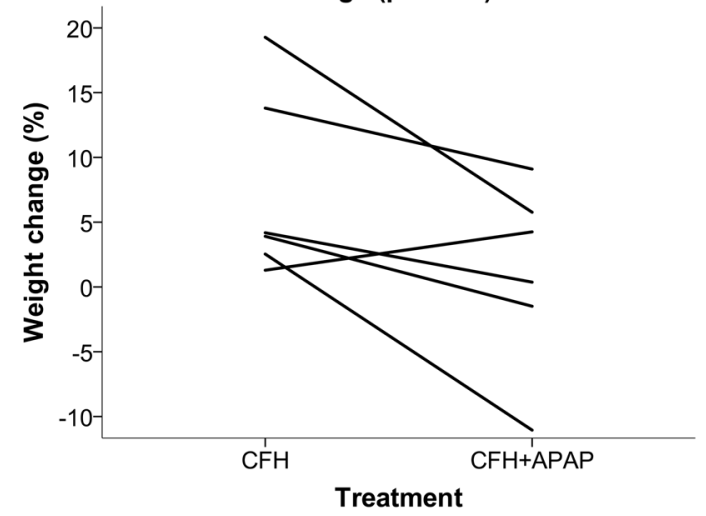

B APAP reduces Evans blue-labeled albumin in paired donor lungs $(p=0.043)$

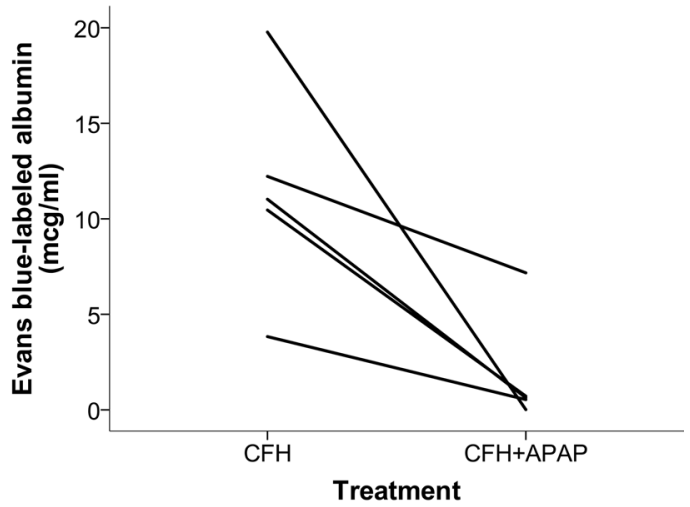

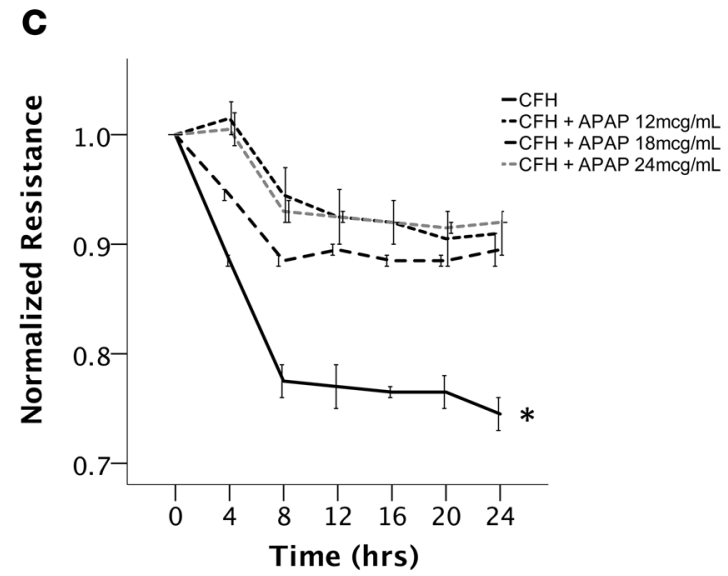

Figure 8. Acetaminophen, a specific hemoprotein reductant, attenuates microvascular permeability caused by cell-free hemoglobin. (A) Ex vivo human isolated perfused lungs inflated with $\mathrm{FiO}_{2} 0.95$ have less weight gain over time after acetaminophen (APAP) therapy compared with cell-free hemoglobin (CFH) alone. Each line connects lung weight change for paired donor lungs subjected to CFH and CFH+APAP. $n=6$ per group, $P=0.046$ by Wilcoxon rank sum testing. (B) Treatment with APAP prevented extravasation of Evans blue-labeled albumin into the airspace. $P=0.043$ by Wilcoxon rank sum testing. (C) APAP prevents CFH-induced permeability as measured by monolayer resistance in human pulmonary microvascular endothelial cells in culture. $n=2-4$ per group, ${ }^{*} P<0.05$ vs. CFH by Mann Whitney $U$ test.

to-donor variability in responses to $\mathrm{CFH}$ in our initial experiments, we tested the effects of hyperoxia or APAP using paired lungs from the same donor. Another potential limitation of our study is that we restricted our observational study to patients with chronic obstructive or fibrotic lung disease as the indication for transplantation. While these diagnoses represent the majority of lung transplant recipients in the US, it is uncertain whether our findings apply to patients with other indications for lung transplantation. It is also possible that other hemoglobin-binding proteins such as haptoglobin, other cell types such as alveolar macrophages, or other antioxidant molecules may alter $\mathrm{CFH}$-mediated vascular injury, and these concepts will require further study.

In summary, this translational study provides both clinical and mechanistic evidence that oxidized $\mathrm{CFH}$ is an important mediator of PGD and offers significant preclinical support for targeting oxidized $\mathrm{CFH}$ for prevention of PGD. The protective effects of APAP both in the isolated perfused human lung and in cultured hPMVECs suggest that clinical trials of perioperative APAP for prevention of PGD after lung transplantation may be warranted.

\section{Methods}

Patient population. Forty cases with grade 3 PGD within 72 hours and 80 control patients with no grade 3 PGD within 72 hours were selected from lung transplant recipients who were prospectively enrolled in the LTOG multicenter observational cohort study (9). Patients were frequency matched for underlying diagnosis and number of lungs transplanted (9). Patients in this study were primarily those recipients with 
COPD or idiopathic pulmonary fibrosis (IPF), the 2 most frequent indications for lung transplantation. No patients received extracorporeal membrane oxygenation (ECMO) prior to lung transplantation. Demographic and clinical information was extracted from the LTOG database.

CFH measurement. Blood was collected into sodium citrate-containing tubes preoperatively and at 6 and 24 hours postoperatively. Samples were centrifuged and plasma collected within 2 hours of sample collection. Plasma CFH was measured by HemoCue (29). The lower limit of detection for this method is $10 \mathrm{mg} / \mathrm{dl}$.

Isolated perfused human lung experiments. Donor lungs that were declined for transplantation were procured from donors managed by Tennessee Donor Services after consent for research was obtained from each donor's next of kin. Lungs were transported to Vanderbilt at $4^{\circ} \mathrm{C}$. For the experimental preparation (Figure 4), lungs were continuously perfused at $37^{\circ} \mathrm{C}$ with DMEM containing $5 \%$ albumin and $16 \%$ (v/v) fresh whole human blood by roller pump to maintain a pulmonary artery pressure of $10-12 \mathrm{mmHg}$ with passive drainage of perfusate through an open left atrium into a reservoir for recirculation $(53,54)$. Lungs were inflated to continuous positive airway pressure (CPAP) of $10 \mathrm{mmHg}$ with either $95 \% \mathrm{O}_{2}\left(\mathrm{FiO}_{2}=0.95\right)$ or $21 \% \mathrm{O}_{2}\left(\mathrm{FiO}_{2}=0.21\right)$, and a recruitment maneuver with sustained inflation to $25 \mathrm{mmHg}$ was performed prior to the experiment $(53,54)$. The lung was suspended from a mass transducer for continuous measurement of lung weight as an index of pulmonary edema formation. Lungs that failed to reach a stable weight over 30-60 minutes after rewarming were discarded. A baseline bronchoalveolar lavage (BAL) was performed with $40 \mathrm{ml}$ normal saline. Then, purified CFH (100 mg/dl, Cell Sciences) or CFH+APAP (15 $\mu \mathrm{g} /$ $\mathrm{ml}$ APAP, MilliporeSigma) was added to the perfusate. After 2 hours, Evans blue-labeled albumin $(0.5 \%$ in $\mathrm{H}_{2} \mathrm{O}$, MilliporeSigma) was added to the perfusate $(15 \mathrm{ml})$ and repeat BAL was performed. Weights are expressed as percent change from baseline. The concentration of Evans blue-labeled albumin in BAL fluid (BALF) was measured spectrophotometrically at $620 \mathrm{~nm}$.

Endothelial permeability assessment. hPMVECs (55) were grown to confluence on 8W10E+ PC electrode arrays (Applied BioPhysics) previously coated with $10 \mu \mathrm{M}$ cysteine and $1 \%$ gelatin. Cells were treated with vehicle control, purified endotoxin-free CFH $(0.25-1.0 \mathrm{mg} / \mathrm{dl})$, or CFH+APAP $(12-24 \mu \mathrm{g} / \mathrm{ml})$ for 24 hours in tissue culture medium (MCDB131 lacking L-glutamine [Thermo Fisher Scientific], supplemented with $10 \mathrm{ng} / \mathrm{ml}$ epidermal growth factor [MilliporeSigma], $1 \mu \mathrm{g} / \mathrm{ml}$ hydrocortisone [MilliporeSigma], $10 \mathrm{mM}$ glutamine [MilliporeSigma], and 10\% FBS [Thermo Fisher Scientific]). Room air $\left(\mathrm{FiO}_{2}=0.21\right)$ or oxygen $\left(\mathrm{FiO}_{2}=0.95\right)$ was bubbled through the media prior to incubation at $37^{\circ} \mathrm{C}$. Paracellular permeability was measured by monitoring monolayer resistance at $4,000 \mathrm{~Hz}$ using electrical cell-substrate impedance sensing (ECIS) (Applied BioPhysics). Data were normalized to the baseline resistance of each chamber prior to addition of treatments. Experiments were performed with $n=2-3$ per group on at least 2 separate days, and representative data are shown.

Statistics. Continuous variables and categorical variables were compared between patient groups using Mann Whitney $U$ or $\chi^{2}$ testing, respectively. Line graphs depict the mean value at each time point with errors bars indicating \pm SEM. Box plots depict the median as a dark horizontal line, the upper and lower edges of the box show the $75 \%$ and $25 \%$ quartiles, respectively, and the whiskers are $\times 1.5$ the IQR. Multivariable logistic regression models were used to determine the effects of confounding variables over the impact of preoperative CFH on risk of PGD grade 3 at 72 hours. The multivariable analysis individually included each variable with $P<0.2$ for an association with PGD in univariate analysis. For this analysis, preoperative $\mathrm{CFH}$ was entered as a categorical variable based on the median value of $<30 \mathrm{mg} / \mathrm{dl}$ or $\geq 30 \mathrm{mg} / \mathrm{dl}$. To assess the contribution of reperfusion $\mathrm{FiO}_{2}$ to the association between $\mathrm{CFH}$ and PGD, the cohort was divided into those with low $(<0.40)$ or high $(\geq 0.40)$ reperfusion $\mathrm{FiO}_{2}$, and a multivariable regression was performed in each group. Lung weight gain and change in EBD concentration over 2 hours was performed by Mann Whitney $U$ testing comparing $\mathrm{CFH}$ with control. Because of the presence of lung donor-specific effects, $\mathrm{CFH}$ in normoxia vs. hyperoxia and $\mathrm{CFH}$ vs. $\mathrm{CFH}+\mathrm{APAP}$ were compared using paired lungs, and analysis was controlled for donor identification by using Wilcoxon signed rank testing. In vitro treatments of cultured endothelial cells were compared by Mann Whitney $U$ testing. $P<0.05$ was considered significant. Statistical analysis was performed using SPSS version 24 (IBM).

Study approval. The study was approved by the Vanderbilt University Medical Center IRB (protocol number 141499). Patients provided informed consent at the time of enrollment into the LTOG study, a multicenter prospective cohort study of patients undergoing lung transplantation designed to evaluate predictors of PGD (5). 


\section{Author contributions}

CMS, JAB, and LBW conceived and designed the study, performed data analysis, and wrote the manuscript. NW, JBM, HN, and AM performed the ex vivo isolated perfused human lung experiments. SRL and JLK performed cultured endothelial cell experiments. JMD and JDC provided clinical information from the LTOG cohort and assisted with manuscript preparation. DJL, SMK, SMP, KMW, AW, VNL, MMC, JBO, PDS, CAH, EC, MKP, GD, and JM are primary investigators in the LTOG cohort study. All authors read and approved the final manuscript.

\section{Acknowledgments}

These studies were funded by NIH HL135849 (LBW, JAB), HL103836 (LBW), HL087115 (JDC), HL126176 (LBW), HL126671 (JAB), HL117676 (JAB), HL121406 (JMD), HL136888 (CMS), Vanderbilt Faculty Research Scholars (CMS), American Thoracic Society (CMS), and the Parker B. Francis Family Foundation (CMS). We would like to thank Tennessee Donor Services for their invaluable assistance in obtaining donor lungs for research. We thank the donor families for their gift to research.

Address correspondence to: Lorraine B. Ware, $116121^{\text {st }}$ Avenue South, Medical Center North, T-1218, Nashville, Tennessee 37232, USA. Phone: 615.322.7872; Email: lorraine.ware@vanderbilt.edu.

1. Christie JD, et al. Report of the ISHLT Working Group on Primary Lung Graft Dysfunction part II: definition. A consensus statement of the International Society for Heart and Lung Transplantation. J Heart Lung Transplant. 2005;24(10):1454-1459.

2. Porteous MK, Diamond JM, Christie JD. Primary graft dysfunction: lessons learned about the first $72 \mathrm{~h}$ after lung transplantation. Curr Opin Organ Transplant. 2015;20(5):506-514.

3. Whitson BA, et al. Risk factors for primary graft dysfunction after lung transplantation. J Thorac Cardiovasc Surg. 2006;131(1):73-80.

4. Kuntz CL, et al. Risk factors for early primary graft dysfunction after lung transplantation: a registry study. Clin Transplant 2009;23(6):819-830.

5. Diamond JM, et al. Clinical risk factors for primary graft dysfunction after lung transplantation. Am J Respir Crit Care Med. 2013;187(5):527-534.

6. Lowery EM, Kuhlmann EA, Mahoney EL, Dilling DF, Kliethermes SA, Kovacs EJ. Heavy alcohol use in lung donors increases the risk for primary graft dysfunction. Alcohol Clin Exp Res. 2014;38(11):2853-2861.

7. Christie JD, et al. Clinical risk factors for primary graft failure following lung transplantation. Chest. 2003;124(4):1232-1241.

8. Liu Y, Liu Y, Su L, Jiang SJ. Recipient-related clinical risk factors for primary graft dysfunction after lung transplantation: a systematic review and meta-analysis. PLoS One. 2014;9(3):e92773.

9. Lederer DJ, et al. Obesity and primary graft dysfunction after lung transplantation: the Lung Transplant Outcomes Group Obesity Study. Am J Respir Crit Care Med. 2011;184(9):1055-1061.

10. Porteous MK, et al. Diastolic Dysfunction Increases the Risk of Primary Graft Dysfunction after Lung Transplant. Am J Respir Crit Care Med. 2016;193(12):1392-1400.

11. Nosotti M, et al. Clinical risk factors for primary graft dysfunction in a low-volume lung transplantation center. Transplant Proc. 2014;46(7):2329-2333.

12. Cantu E, et al. Protein Quantitative Trait Loci Analysis Identifies Genetic Variation in the Innate Immune Regulator TOLLIP in Post-Lung Transplant Primary Graft Dysfunction Risk. Am J Transplant. 2016;16(3):833-840.

13. Somers J, et al. Interleukin-17 receptor polymorphism predisposes to primary graft dysfunction after lung transplantation. $J$ Heart Lung Transplant. 2015;34(7):941-949.

14. Sayah DM, et al. Neutrophil extracellular traps are pathogenic in primary graft dysfunction after lung transplantation. $A m J$ Respir Crit Care Med. 2015;191(4):455-463.

15. Shah RJ, et al. Plasma complement levels are associated with primary graft dysfunction and mortality after lung transplantation. Am J Respir Crit Care Med. 2014;189(12):1564-1567.

16. Diamond JM, Wigfield CH. Role of innate immunity in primary graft dysfunction after lung transplantation. Curr Opin Organ Transplant. 2013;18(5):518-523.

17. Shah RJ, et al. Plasma monocyte chemotactic protein-1 levels at 24 hours are a biomarker of primary graft dysfunction after lung transplantation. Transl Res. 2012;160(6):435-442.

18. Hoffman SA, et al. Plasma cytokines and chemokines in primary graft dysfunction post-lung transplantation. Am J Transplant. 2009;9(2):389-396.

19. Diamond JM, et al. Elevated plasma clara cell secretory protein concentration is associated with high-grade primary graft dysfunction. Am J Transplant. 2011;11(3):561-567.

20. Hashimoto K, et al. Circulating Cell Death Biomarkers May Predict Survival in Human Lung Transplantation. Am J Respir Crit Care Med. 2016;194(1):97-105.

21. Christie JD, et al. Plasma levels of receptor for advanced glycation end products, blood transfusion, and risk of primary graft dysfunction. Am J Respir Crit Care Med. 2009;180(10):1010-1015.

22. Calfee CS, Ware LB. Biomarkers of lung injury in primary graft dysfunction following lung transplantation. Biomark Med. 2007;1(2):285-291.

23. Diamond JM, et al. Elevated plasma angiopoietin-2 levels and primary graft dysfunction after lung transplantation. PLoS One. 
2012;7(12):e51932.

24. Krenn K, Klepetko W, Taghavi S, Lang G, Schneider B, Aharinejad S. Recipient vascular endothelial growth factor serum levels predict primary lung graft dysfunction. Am J Transplant. 2007;7(3):700-706.

25. Christie JD, et al. Association of protein C and type 1 plasminogen activator inhibitor with primary graft dysfunction. $A m J$ Respir Crit Care Med. 2007;175(1):69-74.

26. Suzuki Y, Cantu E, Christie JD. Primary graft dysfunction. Semin Respir Crit Care Med. 2013;34(3):305-319.

27. Diamond JM, et al. The relationship between plasma lipid peroxidation products and primary graft dysfunction after lung transplantation is modified by donor smoking and reperfusion hyperoxia. J Heart Lung Transplant. 2016;35(4):500-507.

28. Janz DR, et al. Randomized, placebo-controlled trial of acetaminophen for the reduction of oxidative injury in severe sepsis: the Acetaminophen for the Reduction of Oxidative Injury in Severe Sepsis trial. Crit Care Med. 2015;43(3):534-541.

29. Janz DR, et al. Association between, acetaminophen, and mortality in patients with sepsis: an observational study. Crit Care Med. 2013;41(3):784-790.

30. Bastarache JA, et al. Low levels of tissue factor lead to alveolar haemorrhage, potentiating murine acute lung injury and oxidative stress. Thorax. 2012;67(12):1032-1039.

31. Shaver CM, et al. Cell-free hemoglobin: a novel mediator of acute lung injury. Am J Physiol Lung Cell Mol Physiol. 2016;310(6):L532-L541.

32. Vermeulen Windsant IC, Hanssen SJ, Buurman WA, Jacobs MJ. Cardiovascular surgery and organ damage: time to reconsider the role of hemolysis. J Thorac Cardiovasc Surg. 2011;142(1):1-11.

33. Brittain EL, et al. Elevation of plasma cell-free hemoglobin in pulmonary arterial hypertension. Chest. 2014;146(6):1478-1485

34. Reeder BJ, Wilson MT. Hemoglobin and myoglobin associated oxidative stress: from molecular mechanisms to disease States. Curr Med Chem. 2005;12(23):2741-2751.

35. Boutaud $\mathrm{O}$, et al. Acetaminophen inhibits hemoprotein-catalyzed lipid peroxidation and attenuates rhabdomyolysis-induced renal failure. Proc Natl Acad Sci USA. 2010;107(6):2699-2704.

36. Rusak T, Misztal T, Piszcz J, Tomasiak M. Nitric oxide scavenging by cell-free hemoglobin may be a primary factor determining hypertension in polycythemic patients. Free Radic Res. 2014;48(2):230-238.

37. Ouellet M, Percival MD. Mechanism of acetaminophen inhibition of cyclooxygenase isoforms. Arch Biochem Biophys. 2001;387(2):273-280.

38. Schaer DJ, Buehler PW. Cell-free hemoglobin and its scavenger proteins: new disease models leading the way to targeted therapies. Cold Spring Harb Perspect Med. 2013;3(6):a013433.

39. Risbano MG, et al. Effects of Aged Stored Autologous Red Blood Cells on Human Endothelial Function. Am J Respir Crit Care Med. 2015;192(10):1223-1233.

40. Rother RP, Bell L, Hillmen P, Gladwin MT. The clinical sequelae of intravascular hemolysis and extracellular plasma hemoglobin: a novel mechanism of human disease. JAMA. 2005;293(13):1653-1662.

41. Lisk C, et al. Hemoglobin-induced endothelial cell permeability is controlled, in part, via a myeloid differentiation primary response gene-88-dependent signaling mechanism. Am J Respir Cell Mol Biol. 2013;49(4):619-626.

42. Silva G, Jeney V, Chora A, Larsen R, Balla J, Soares MP. Oxidized hemoglobin is an endogenous proinflammatory agonist that targets vascular endothelial cells. J Biol Chem. 2009;284(43):29582-29595.

43. Potor L, et al. Atherogenesis may involve the prooxidant and proinflammatory effects of ferryl hemoglobin. Oxid Med Cell Longev. 2013;2013:676425.

44. Baldwin AL. Modified hemoglobins produce venular interendothelial gaps and albumin leakage in the rat mesentery. Am $J$ Physiol. 1999;277(2 Pt 2):H650-H659.

45. Godzich M, et al. Activation of the stress protein response prevents the development of pulmonary edema by inhibiting VEGF cell signaling in a model of lung ischemia-reperfusion injury in rats. FASEB J. 2006;20(9):1519-1521.

46. Krenn K, Klepetko W, Taghavi S, Paulus P, Aharinejad S. Vascular endothelial growth factor increases pulmonary vascular permeability in cystic fibrosis patients undergoing lung transplantation. Eur J Cardiothorac Surg. 2007;32(1):35-41.

47. Su G, et al. Integrin alphavbeta5 regulates lung vascular permeability and pulmonary endothelial barrier function. Am J Respir Cell Mol Biol. 2007;36(3):377-386.

48. Su G, et al. Effective treatment of mouse sepsis with an inhibitory antibody targeting integrin $\alpha v \beta 5$. Crit Care Med. 2013;41(2):546-553.

49. Mallavia B, Liu F, Sheppard D, Looney MR. Inhibiting Integrin $\alpha v \beta 5$ Reduces Ischemia-Reperfusion Injury in an Orthotopic Lung Transplant Model in Mice. Am J Transplant. 2016;16(4):1306-1311.

50. Ben Moussa S, Sfaxi I, Tabka Z, Ben Saad H, Rouatbi S. Oxidative stress and lung function profiles of male smokers free from COPD compared to those with COPD: a case-control study. Libyan J Med. 2014;9:23873.

51. Ware LB, et al. Donor smoking is associated with pulmonary edema, inflammation and epithelial dysfunction in ex vivo human donor lungs. Am J Transplant. 2014;14(10):2295-2302.

52. Ware LB, et al. Assessment of lungs rejected for transplantation and implications for donor selection. Lancet. 2002;360(9333):619-620.

53. Frank JA, Briot R, Lee JW, Ishizaka A, Uchida T, Matthay MA. Physiological and biochemical markers of alveolar epithelial barrier dysfunction in perfused human lungs. Am J Physiol Lung Cell Mol Physiol. 2007;293(1):L52-L59.

54. Lee JW, Fang X, Gupta N, Serikov V, Matthay MA. Allogeneic human mesenchymal stem cells for treatment of E. coli endotoxin-induced acute lung injury in the ex vivo perfused human lung. Proc Natl Acad Sci USA. 2009;106(38):16357-16362.

55. Fessel JP, et al. Metabolomic analysis of bone morphogenetic protein receptor type 2 mutations in human pulmonary endothelium reveals widespread metabolic reprogramming. Pulm Circ. 2012;2(2):201-213. 